\title{
EXPONENTIAL SYNCHRONIZATION OF FINITE-DIMENSIONAL KURAMOTO MODEL AT CRITICAL COUPLING STRENGTH*
}

\author{
YOUNG-PIL CHOI ${ }^{\dagger}$, SEUNG-YEAL HA \\ MYUNGJOO KANG
}

\begin{abstract}
We discuss the exponential synchronization for an ensemble of Kuramoto oscillators at the critical coupling strength, which is the diameter of the set consisting of natural frequencies. When the number of distinct natural frequencies is greater than two and the initial phases are strictly confined in an interval of length $\frac{\pi}{2}$, we show that the initial configuration evolves toward a phase-locked state at least exponentially fast. This fast convergence toward the phase-locked state is markedly different from an ensemble of Kuramoto oscillators with only two distinct natural frequencies. For this, we derive a Gronwall inequality for the frequency diameter to obtain complete synchronization. We also compare our analytical results with numerical simulation results.
\end{abstract}

Key words. Critical coupling strength, exponential synchronization, Kuramoto model, natural frequency.

AMS subject classifications. 92D25, 74A25, 76N10.

\section{Introduction}

The objective of this paper is to present an exponential synchronization for an ensemble of Kuramoto oscillators, when the coupling strength is exactly equal to the diameter of the natural frequencies. The synchronization of large weakly coupled oscillators appears in many biological complex systems, e.g., metabolic synchrony in yeast cell suspension, synchronous firing of a cardiac pacemaker, and flashing of fireflies (see $[2,3,10,19,20,29,30]$ for details). Mathematical studies of the synchronization of coupled oscillators were initiated by two pioneers, Winfree and Kuramoto, about 40 years ago. The emergence of engineering applications based on complex networks such as power system networks and multi-agent unmanned aerial vehicles [8, 9, 14] has increased the interest in Kuramoto type models in several scientific areas. Indeed, the Kuramoto model has been studied extensively in many disciplines such as applied mathematics, control theory, and statistical physics.

Kuramoto oscillators can be conceived as active point rotors moving on the unit circle $\mathbb{S}^{1}$, and the Kuramoto phase model is a system of ODEs that is weakly coupled via sinusoidal coupling. More precisely, we denote $x_{i}:=e^{\sqrt{-1} \theta_{i}}, \theta_{i} \in \mathbb{R}$ as the position of the $i$-th rotor in $\mathbb{C}$. In this case, the phase dynamics is governed by the following system of ODEs $[15,16]$ in $\mathbb{R}^{N}$ :

$$
\dot{\theta}_{i}=\Omega_{i}+\frac{K}{N} \sum_{j=1}^{N} \sin \left(\theta_{j}-\theta_{i}\right), \quad t>0, \quad i=1, \ldots, N,
$$

${ }^{*}$ Received: December 23, 2011; accepted (in revised form): June 12, 2012. Communicated by Paul Milewski.

${ }^{\dagger}$ Department of Mathematical Sciences, Seoul National University, Seoul 151-747, Korea (freelyer@snu.ac.kr).

${ }^{\ddagger}$ Department of Mathematical Sciences, Seoul National University, Seoul 151-747, Korea (syha@snu.ac.kr), http://www.math.snu.ac.kr/ ${ }^{\sim}$ syha

$\S$ Department of Mathematical Sciences, Seoul National University, Seoul 151-747, Korea (wjdjr1@snu.ac.kr).

『Department of Mathematical Sciences, Seoul National University, Seoul 151-747, Korea (mkang@ snu.ac.kr). 
given the initial data:

$$
\theta_{i}(0)=\theta_{i 0}
$$

where $\Omega_{i}, K$, and $N$ are the intrinsic natural frequency of the $i$-th oscillator drawn from some distribution function $g=g(\Omega)$, the uniform positive coupling strength, and the size of the system, respectively. If necessary, we can take a rotating frame and assume that

$$
\sum_{i=1}^{N} \Omega_{i}=0
$$

Note that if $\theta=\left(\theta_{1}, \cdots, \theta_{N}\right)$ is a solution, then $\theta+\alpha, \alpha \in \mathbb{Z}^{N}$ is a solution due to the translation invariance and periodicity of the coupling function. Thus, the system (1.1) naturally induces a dynamical system for $N$-tori $\mathbb{T}^{N}$.

Next, we briefly review the published literature on synchronization estimates for the Kuramoto model. The Kuramoto model (1.1) and its variants have been derived heuristically from the complex Ginzburg-Landau equation [16] and as an averaged system of the coupled system of Josephson junction arrays [28]. Ermentrout [10] found a critical coupling where all oscillators become phase-locked, which was independent of the number of oscillators. The stability of the phase-locked state was established by van Hemmen and Wreszinski [25] for large coupling using the Lyapunov functional approach, and Jadbabaie et al. [14] also considered the stability of the phase-locked state on general connectivity graphs and derived the computable bounds for the critical coupling constant using tools from spectral graph theory. The linear stability of the phase-locked state and rigorous characterization of the spectrum for the Kuramoto model were treated by Mirollo and Strogatz [17, 18, 23]. We refer to [28] for a treatment of the synchronization of super-conducting Josephson junction arrays. For a detailed description of the Kuramoto model (1.1), we refer to survey papers [1, 3, 22]. We now discuss the synchronization problem. In [16], Kuramoto introduced an order parameter $r^{\infty}=r^{\infty}(K)$ to measure the degree of synchronization:

$$
r(K, N, t)=\left|\frac{1}{N} \sum_{j=1}^{N} e^{i \theta_{j}(t)}\right|, \quad r^{\infty}(K):=\lim _{t \rightarrow \infty} \lim _{N \rightarrow \infty} r(K, N, t) .
$$

Note that $r=0$ and 1 denote the completely incoherent state and complete phase synchronization, respectively. Kuramoto also showed that there is a critical coupling strength $K_{c}$ such that $r^{\infty}$ bifurcates from zero to non-zero values as $K$ exceeds $K_{c}$, i.e., the transition from "the completely incoherent state" to "a partially coherent state" in the thermodynamic limit $(N \rightarrow \infty)$. Indeed, the critical coupling strength $K_{c}$ can be explicitly computable for unimodal and symmetric distributions $g$. However, for a finite-dimensional case with $N<\infty$, the identification of such a critical value is unclear because the fluctuation of the order parameter is $\mathcal{O}\left(N^{-\frac{1}{2}}\right)$ (see $[1,22]$ and references therein). Of course, we may still argue that the critical value is close to $K_{c}$ when $N$ is sufficiently large.

In this paper, we are interested in the complete synchronization problem where all oscillators are phase-locked asymptotically. As observed in $[5,8,11]$, for a finitedimensional Kuramoto model (1.1) with $N<\infty$, a new threshold coupling strength $K=D(\Omega)$ appears, when the complete frequency synchronization (in short CFS) of (1.1) with $N<\infty$ is considered (see Definition 2.1). In fact, Dörfler and Bullo [8] 
showed that if $K \leq D(\Omega)$, then there exist a set of natural frequencies $\left\{\Omega_{1}, \cdots \Omega_{N}\right\}$ and initial configuration $\theta_{0}$,

$$
D(\Omega)=\Omega_{M}-\Omega_{m} \quad \text { and } \quad D\left(\theta_{0}\right)<\pi
$$

which do not lead to a phase-locked state exponentially fast. In contrast, when $K>$ $D(\Omega)$, there exists $\gamma \in\left(\frac{\pi}{2}, \pi\right]$ such that the system (1.1) synchronizes exponentially fast for all possible distributions of the natural frequencies whose diameters are equal to $D(\Omega)$ and for all initial phases $\theta_{0}$ with $D\left(\theta_{0}\right)<\gamma$. In this sense, the strength $K=$ $D(\Omega)$ plays the role of a threshold from "partially synchronized states" to "completely synchronized states" in the dynamics of (1.1). Thus, throughout the paper, we use the terminologies "super-critical" and "critical" regimes to denote the regimes $K>D(\Omega)$ and $K=D(\Omega)$, respectively. In $[4,5,6,8,9,11,13]$, the CFS was extensively studied in the super-critical regime $K>D(\Omega)$. In contrast, the corresponding synchronization problem has not been well studied in the critical regime where $K=D(\Omega)$. In [12], Ha and Kang considered the special case where only two distinct natural frequencies are present in the set of natural frequencies, i.e., a mixed ensemble of two ensembles of identical oscillators, and the initial configuration is confined in an arc with length $\frac{\pi}{2}$. In this situation, the phase configurations are confined within an arc with length $\frac{\pi}{2}$ and the complete frequency states emerge algebraically slowly in the order of $\mathcal{O}(1)(1+$ $t)^{-1}$. Thus it is natural to ask the following questions:

- Given an ensemble of nonidentical Kuramoto oscillators with at least three distinct natural frequencies, is it possible to have complete frequency synchronization asymptotically with the critical coupling $K=D(\Omega)$ ?

- If so, what will be the convergence rate toward complete synchronization? Is it still algebraic?

The main contributions of this paper are answers to the questions posed above regarding the CFS for the Kuramoto model (1.1) where we provide quantitative estimates. Our main result can be summarized as follows. When the number of distinct natural frequencies is greater than two, and the Kuramoto oscillators are strictly confined in the arc with a length $\frac{\pi}{2}$, the Kuramoto oscillators will approach the complete frequency states at least exponentially fast. This fast relaxation is markedly different from the special case [12], where a slow relaxation of the two natural frequencies is observed.

The rest of the paper is organized as follows. In Section 2, we briefly outline our main results on CFS at the critical coupling strength. In Section 3, we present two basic estimates for the pointwise estimate of phase differences and the existence of invariant regions given the dynamics of (1.1). In Section 4, we present a proof of Theorem 2.2 and in Section 5 we provide several numerical simulations, which we compare with our analytical results. Finally, Section 6 provides a summary of our main results. In Appendix A, we list several elementary estimates.

Notation: Next, we provide several notations that are used throughout the paper.

$$
\begin{array}{ll}
\ell(\Omega):=\text { The number of distinct natural frequencies, } & \omega_{i}=\dot{\theta}_{i}, \\
D(\theta):=\max _{1 \leq i, j \leq N}\left|\theta_{i}-\theta_{j}\right|, \quad D(\omega):=\max _{1 \leq i, j \leq N}\left|\omega_{i}-\omega_{j}\right|, & D(\Omega):=\max _{1 \leq i, j \leq N}\left|\Omega_{i}-\Omega_{j}\right| .
\end{array}
$$




\section{Discussion of the main result}

In this section, we briefly outline the main result of this paper. The well-posedness issue of the Kuramoto model (1.1) is standard because the right hand side of (1.1) is globally Lipschitz continuous with respect to the state variables. Thus, one of the interesting issues for (1.1) is the emergence of collective motion "synchronization" that depends on the distribution of the natural frequency and the coupling strength. Under the Assumption (1.3), the phase-locked states for (1.1) correspond to the equilibrium solutions:

$$
\Omega_{i}+\frac{K}{N} \sum_{j=1}^{N} \sin \left(\theta_{j}-\theta_{i}\right)=0, \quad i=1, \cdots, N .
$$

It is easy to see that if $\left|\Omega_{i}\right|>K$ for some $i$, then the system (2.1) does not permit equilibrium solutions, because we have

$$
\left|\Omega_{i}+\frac{K}{N} \sum_{j=1}^{N} \sin \left(\theta_{j}-\theta_{i}\right)\right| \geq\left|\Omega_{i}\right|-K>0 .
$$

Thus, to find the phase-locked solution for (1.1)-(1.3), the coupling strength $K$ should be sufficiently large. It is natural to ask how large the coupling strength should be, which is a question of "computing the critical coupling strength". This question has been discussed in $[8,9,26,27]$. Next, we restate the definition of complete frequency synchronization (CFS) as follows.

Definition 2.1. [11] The Kuramoto model (1.1) has an asymptotically complete (frequency) synchronization if and only if the relative frequency differences converge to zero as $t \rightarrow \infty$ :

$$
\lim _{t \rightarrow \infty}\left|\dot{\theta}_{i}(t)-\dot{\theta}_{j}(t)\right|=0, \quad \text { for all } \quad i, j \in\{1, \ldots, N\} .
$$

In [8], Dörfler and Bullo reviewed several synchronization conditions found in the literature and provided a necessary and sufficient condition for the super-critical case $K>D(\Omega)$ for asymptotic exponential synchronization with respect to the arbitrary natural frequencies drawn from the interval $\left[-\frac{D(\Omega)}{2}, \frac{D(\Omega)}{2}\right]$. In this case, they also showed that the initial phase configuration $\theta_{0}$ that belongs to $\mathcal{S}$,

$$
\mathcal{S}:=\left\{\theta \in \mathbb{R}^{N}: D(\theta)<\sin ^{-1}\left(\frac{D(\Omega)}{K}\right) \in\left(\frac{\pi}{2}, \pi\right)\right\},
$$

leads to a phase-locked state at least exponentially rapidly. Note that this set $\mathcal{S}$ certainly contains our admissible set $\mathcal{R}$.

Recall that the purpose of this paper is to consider the CFS at the critical coupling strength $K=D(\Omega)$. Therefore, our result is complementary to that of [8]. We now describe our framework $(\mathcal{F})$ and main results in Section 4.

- $(\mathcal{F} 1)$ : A system of Kuramoto oscillators consists of many heterogeneous subgroups:

$$
\ell(\Omega) \geq 3 .
$$

- $(\mathcal{F} 2)$ : The coupling strength $K$ is critical:

$$
K=D(\Omega) .
$$


Under the above framework $(\mathcal{F})$, the CFS at a critical coupling strength can be stated as follows.

THEOREM 2.2. Suppose that the framework $(\mathcal{F})$ holds, and let $\theta=\theta(t)$ be the solution to the system (1.1) - (1.3) with the initial data $\theta_{0}$ :

$$
\mathcal{R}:=\left\{\theta \in \mathbb{R}^{N}: 0<\theta_{i}<\frac{\pi}{2}, \quad i=1, \cdots, N\right\} .
$$

Thus, CFS occurs asymptotically and we also have

(i) $D_{\theta}^{\infty}:=\sup _{t \geq 0} D(\theta(t))<\frac{\pi}{2}$,

(ii) $D(\omega(t)) \leq D\left(\omega_{0}\right) \exp \left[-K\left(\cos D_{\theta}^{\infty}\right) t\right], \quad t \geq 0, \quad \omega_{0}:=\dot{\theta}(0)$.

REMARK 2.1.

1. For the supercritical case $K>D(\Omega)$, the synchronization estimates of the system (1.1) were studied in $[4,5]$. For the mean-field case $(N \rightarrow \infty)$, the linearized stability of the phase-locked state was investigated in $[17,18,23,24]$.

2. For $\ell(\Omega)=2$ and $K=D(\Omega)$, it is known that the speed of complete frequency synchronization can be algebraic for some class of initial configurations with the order $\mathcal{O}(1)(1+t)^{-1}$. Thus, the dynamic feature of $\ell(\Omega) \geq 3$ is essentially different from that of $\ell(\Omega)=2$.

\section{Existence of a positively invariant set}

In this section, we present a proof of the positive invariance of the set $\mathcal{R}$ under the Kuramoto flow (1.1), which will be crucial in the proof of Theorem 2.2 in next section.

Before we study the invariance of the set $\mathcal{R}$ in (2.2), we recall some estimates of the phase differences from [5]. We set

$$
\begin{aligned}
\theta_{i j} & :=\theta_{i}-\theta_{j}, \quad \Omega_{i j}:=\Omega_{i}-\Omega_{j}, \\
E_{i j}^{l} & :=1-\frac{\cos \left(\frac{\theta_{l i}+\theta_{l j}}{2}\right)}{\cos \left(\frac{\theta_{j i}}{2}\right)}, \quad \beta_{i j}(N, \theta):=1-\frac{1}{N} \sum_{l \neq i, j} E_{i j}^{l} .
\end{aligned}
$$

Proposition 3.1. Suppose that the framework $(\mathcal{F})$ holds, and let $\theta=\theta(t)$ be the solution to the system (1.1) - (1.3) with initial data $\theta_{0} \in \mathcal{R}$. Then, the following estimates hold:

(i) The phase difference $\theta_{i j}$ satisfies

$$
\dot{\theta}_{i j}+K \beta_{i j}(N, \theta) \sin \theta_{i j}=\Omega_{i j} .
$$

(ii) Suppose that the pair $(i, j)$ satisfies

$$
\theta_{i}\left(t_{0}\right)>\theta_{j}\left(t_{0}\right) \quad \text { and } \quad \Omega_{i} \geq \Omega_{j} \text { for some } t_{0} \in \mathbb{R}_{+} .
$$

Then, the $i$ and $j$-oscillators will not meet after $t=t_{0}$, i.e.,

$$
\theta_{i}(t)>\theta_{j}(t), \quad t>t_{0} .
$$


(iii) If $\Omega_{i}>\Omega_{j}$, then there exists a positive time $t_{i j}^{*}$ such that

$$
\theta_{i}(t)>\theta_{j}(t) \quad \text { for } \quad t \geq t_{i j}^{*} \text {. }
$$

Proof. The corresponding estimates in [5] are based on the assumption $K>$ $D(\Omega)$. However, the straightforward modification in [5] still applies in the critical case $K=D(\Omega)$. Therefore, we omit its proof.

REMARK 3.1.

1. The results in Proposition 3.1 imply that the oscillators will be arranged according to the size of their eventual natural frequency, so the collision times are less than or equal to $N(N-1) / 2$, since we can take $t_{i j}^{*}$ to be a time after the collision time between two different oscillators and these oscillators do not collide for $t>t_{i j}^{*}$.

2. From [5], we can obtain upper and lower bounds for the mean field coupling term $\beta_{i j}$ :

$$
L(N) \leq \beta_{i j}(N, \theta) \leq U(N)
$$

where $L(N)$ and $U(N)$ are explicitly given by

$$
U(N):=\frac{2}{N}+\sqrt{2}\left(1-\frac{2}{N}\right), \quad L(N):=\frac{2}{N} .
$$

By combining the estimates (3.1) and (3.2), when two oscillators $i$ and $j$ have the same natural frequencies, their phase difference $\theta_{i j}$ can be estimated as follows:

$$
\theta_{i j}(0) e^{-K U(N) t} \leq \theta_{i j}(t) \leq \theta_{i j}(0) e^{-K L(N) \frac{2}{\pi} t}, \quad t \geq 0 .
$$

The above lower bound estimate implies that there will be no finite time phase collisions between two identical oscillators with different initial phases. More precisely, if $\theta_{i 0} \neq \theta_{j 0}$, then the $i$-th and $j$-th identical oscillators never meet during finite time. We can also see that if $\Omega_{i j}=0$, then

$$
\theta_{i j}(t) \rightarrow 0 \quad \text { as } \quad t \rightarrow \infty
$$

based on the upper bound estimate.

Let $\Omega_{i_{1}}, \cdots \Omega_{i_{\ell}}$ be the distinct natural frequencies, and $\left\{\mathcal{C}_{1}, \ldots, \mathcal{C}_{\ell}\right\}$ are the partition of the set $\{1, \cdots, N\}$ such that

$$
\mathcal{C}_{k}:=\left\{j: \Omega_{j}=\Omega_{i_{k}}\right\} .
$$

Lemma 3.2. Let $\theta=\theta(t)$ be the solution to the system (1.1) - (1.3) with a uniform bound:

$$
\sup _{t \geq 0} \max _{1 \leq i \leq N}\left|\theta_{i}(t)\right| \leq \frac{\pi}{2}
$$

Then, there exist sequences $\left\{\tau_{k}\right\}, \omega_{i}^{\infty}$, and $\theta_{i}^{\infty}$ such that

(i) $\lim _{k \rightarrow \infty} \omega_{i}\left(\tau_{k}\right)=\omega_{i}^{\infty}, \quad \lim _{k \rightarrow \infty} \theta_{i}\left(\tau_{k}\right)=\theta_{i}^{\infty}, \quad$ for all $i \in\{1, \ldots, N\}$,

(ii) $\lim _{t \rightarrow \infty} \theta_{i}=\lim _{t \rightarrow \infty} \theta_{j} \quad$ for all $i, j \in \mathcal{C}_{k}, \quad k \in\{1, \cdots, \ell\}$, 
where $\theta_{i}^{\infty}$ is a nonnegative constant in the interval $\left[0, \frac{\pi}{2}\right]$.

Proof.

(i) We split this proof into two steps.

- Step A (Construction of limit points $\left\{\omega_{i}^{\infty}\right\}$ ): Note that the set of frequency of the first oscillator $\left\{\omega_{1}(t)\right\}$ is uniform bounded

$$
\left|\omega_{1}(t)\right| \leq\left|\Omega_{1}\right|+K
$$

According to the Bolzano-Weierstrass Theorem, we can choose a sequence $\left\{t_{k}^{1}\right\}$ such that

$$
\lim _{k \rightarrow \infty} \omega_{1}\left(t_{k}^{1}\right)=\omega_{1}^{\infty} \quad \text { for some } \omega_{1}^{\infty} .
$$

Since the set $\left\{\omega_{2}\left(t_{k}^{1}\right)\right\}$ is uniformly bounded, we have

$$
\left|\omega_{2}\left(t_{k}^{1}\right)\right| \leq\left|\Omega_{2}\right|+K
$$

Again, according to the Bolzano-Weierstrass theorem, we can choose a subsequence $\left\{t_{k}^{2}\right\}$ of $\left\{t_{k}^{1}\right\}$ such that

$$
\lim _{k \rightarrow \infty} \omega_{1}\left(t_{k}^{2}\right)=\omega_{2}^{\infty}
$$

In a similar manner, we can choose a subsequence $\left\{t_{k}^{N}\right\}$ of $\left\{t_{k}^{N-1}\right\}$ such that

$$
\lim _{k \rightarrow \infty} \dot{\theta}_{N}\left(t_{k}^{N}\right)=\omega_{N}^{\infty}
$$

- Step B (Construction of limit points $\left\{\theta_{i}^{\infty}\right\}$ ): Based on a uniform boundedness assumption, we know that the $\theta_{i}$ is bounded so we can apply the above iterative argument to the $\theta_{i}$ again. Thus, we obtain that there is a subsequence $\left\{t_{k}^{N, N}\right\}$ of $\left\{t_{k}^{N, N-1}\right\}$ such that

$$
\lim _{k \rightarrow \infty} \theta_{i}\left(t_{k}^{N, N}\right)=\theta_{i}^{\infty} \quad \text { where } \quad \theta_{i}^{\infty} \in\left[0, \frac{\pi}{2}\right]
$$

Finally, we set

$$
\tau_{k}:=t_{k}^{N, N}
$$

Therefore, we have

$$
\lim _{k \rightarrow \infty} \omega_{i}\left(\tau_{k}\right)=\omega_{i}^{\infty} \quad \text { and } \quad \lim _{k \rightarrow \infty} \theta_{i}\left(\tau_{k}\right)=\theta_{i}^{\infty} .
$$

(ii) Thanks to Remark 3.1 (2), if $i, j \in \mathcal{C}_{k}$ for some $k \in\{1, \ldots, \ell\}$, we have

$$
\lim _{t \rightarrow \infty} \theta_{i}(t)=\lim _{t \rightarrow \infty} \theta_{j}(t)
$$

This yields the desired estimate (ii).

We now return to the positive invariance of the set $\mathcal{R}$ in the following proposition. 
Proposition 3.3. Suppose that the framework $(\mathcal{F})$ holds, and let $\theta=\theta(t)$ be the solution to the system (1.1) - (1.3) with initial data $\theta_{0} \in \mathcal{R}$. Then we have

$$
D_{\theta}^{\infty}:=\sup _{t \geq 0} D(\theta(t))<\frac{\pi}{2} \text {. }
$$

Proof. The proof is rather lengthy, so we split it into two parts. In Part A, we show that $D_{\theta}^{\infty} \in\left[0, \frac{\pi}{2}\right]$, and in Part B we show that $D_{\theta}^{\infty}<\frac{\pi}{2}$.

Without loss of generality, we can assume that the initial phase configurations and natural frequencies are ordered according to Proposition 3.1:

$$
0<\theta_{N 0} \leq \cdots \leq \theta_{20} \leq \theta_{10}<\frac{\pi}{2}, \quad \Omega_{N} \leq \cdots \leq \Omega_{2} \leq \Omega_{1}, \quad N \in \mathcal{C}_{l}, \quad 1 \in \mathcal{C}_{1} .
$$

- Part A (Rough estimate): We claim

$$
D(\theta(t))<\frac{\pi}{2}, \quad t \geq 0
$$

This implies that

$$
D_{\theta}^{\infty} \leq \frac{\pi}{2}
$$

We then apply proof by contradiction. We set

$$
\Gamma:=\left\{t \in[0, \infty): D(\theta(t))<\frac{\pi}{2}\right\}, \quad T_{*}:=\sup \Gamma .
$$

Note that since $0 \in \Gamma$ and $D(\theta(t))$ is continuous, the set contains some small interval $[0, \varepsilon)$ for a small positive constant $0<\varepsilon \ll 1$.

We claim that:

$$
T_{*}=\infty \text {. }
$$

The proof of claim: Suppose not, i.e., $T_{*}<\infty$. Since $D(\theta(t))$ is continuous, we should have

$$
\lim _{t \rightarrow T_{*}-} D(\theta(t))=\frac{\pi}{2}
$$

We set

$$
\theta_{M}:=\max _{1 \leq i \leq N} \theta_{i}, \quad \theta_{m}:=\min _{1 \leq i \leq N} \theta_{i} .
$$

Next, we estimate the maximal and minimal fluctuations separately.

Case A (Maximal phase fluctuation): Since $\theta_{M}$ is Lipschitz continuous, it is differentiable almost everywhere in time $t$. Furthermore, we can show that the nondifferentiable points are countable and isolated based on the same argument stated in [11]. Thus, there exists at most a countable number of times $0:=t_{0}<t_{1}<\cdots<t_{\infty}=T_{*}$ such that

$$
\theta_{M} \text { is differentiable in the time interval }\left(t_{k-1}, t_{k}\right), k=1,2, \cdots .
$$

We now use

$$
\sin x \leq \frac{2}{\pi} x, \quad x \in\left[-\frac{\pi}{2}, 0\right]
$$




$$
-\frac{\pi}{2} \leq-D(\theta(t)) \leq \theta_{i}(t)-\theta_{M}(t) \leq 0, \quad \text { a.e. } t \in\left[0, T_{*}\right),
$$

to derive a differential inequality:

$$
\begin{aligned}
\dot{\theta}_{M} & =\Omega_{M}+\frac{K}{N} \sum_{j=1}^{N} \sin \left(\theta_{j}-\theta_{M}\right) \\
& \leq \Omega_{M}+\frac{2 K}{N \pi} \sum_{j=1}^{N}\left(\theta_{j}-\theta_{M}\right), \quad t \in\left(t_{k-1}, t_{k}\right) .
\end{aligned}
$$

Case B (Minimal fluctuation): We use the same argument from Case A to find

$$
\dot{\theta}_{m} \geq \Omega_{m}+\frac{2 K}{N \pi} \sum_{j=1}^{N}\left(\theta_{j}-\theta_{m}\right), \quad t \in\left(t_{k-1}, t_{k}\right) .
$$

We now combine Case A-Case B to yield

$$
\dot{D}(\theta(t)) \leq D(\Omega)-\frac{2 K}{\pi} D(\theta(t))=D(\Omega)-\frac{2 D(\Omega)}{\pi} D(\theta(t)), \quad \text { a.e. } t,
$$

and we use the continuity of $D(\theta(t))$ to obtain

$$
\begin{aligned}
D(\theta(t)) & \leq D\left(\theta_{0}\right) e^{-\frac{2 D(\Omega) t}{\pi}}+\frac{\pi}{2}\left[1-e^{-\frac{2 D(\Omega) t}{\pi}}\right] \\
& =\frac{\pi}{2}+\left(D\left(\theta_{0}\right)-\frac{\pi}{2}\right) e^{-\frac{2 D(\Omega) t}{\pi}}
\end{aligned}
$$

In (3.4), we take $t \rightarrow T_{*}-$ and obtain

$$
\lim _{t \rightarrow T_{*}-} D(\theta(t)) \leq \frac{\pi}{2}+\left(D\left(\theta_{0}\right)-\frac{\pi}{2}\right) e^{-\frac{2 D(\Omega) T_{*}}{\pi}}<\frac{\pi}{2} .
$$

This is contradictory to (3.3). Therefore, we have

$$
T_{*}=\infty, \quad D(\theta(t))<\frac{\pi}{2}, \quad t \geq 0 .
$$

- Part B (Refined estimate): We claim that

$$
\sup _{t \geq 0} D(\theta(t))=\frac{\pi}{2} \quad \text { does not hold. }
$$

Suppose it holds, so that a sequence $\left\{t_{n}\right\}$ exists that satisfies

$$
\lim _{n \rightarrow \infty} D\left(\theta\left(t_{n}\right)\right)=\frac{\pi}{2}
$$

By the Assumption (3.5) and Lemma 3.2, there exists a subsequence $\left\{t_{n_{k}}\right\}$ of $\left\{t_{n}\right\}$ such that for all $i \in\{1, \ldots, N\}$

$$
\lim _{k \rightarrow \infty} \dot{\theta}_{i}\left(t_{n_{k}}\right)=\omega_{i}^{\infty} \text { and } \lim _{k \rightarrow \infty} \theta_{i}\left(t_{n_{k}}\right)=\theta_{i}^{\infty},
$$

and

$$
\theta_{i}^{\infty}=\theta_{j}^{\infty} \quad \text { for all } \quad i, j \in \mathcal{C}_{k}
$$


where $\theta_{i}^{\infty} \in\left[0, \frac{\pi}{2}\right]$. We set

$$
\tilde{\theta}_{k}^{\infty}:=\theta_{i}^{\infty} \quad \text { for all } \quad i \in \mathcal{C}_{k} .
$$

In particular, we have

$$
\lim _{k \rightarrow \infty} \theta_{1}\left(t_{n_{k}}\right)=\frac{\pi}{2} \quad \text { and } \quad \lim _{k \rightarrow \infty} \theta_{N}\left(t_{n_{k}}\right)=0 .
$$

From Part A, we also have

$$
\lim _{k \rightarrow \infty} \dot{\theta}_{1}\left(t_{n_{k}}\right) \geq 0 \quad \text { and } \quad \lim _{k \rightarrow \infty} \dot{\theta}_{N}\left(t_{n_{k}}\right) \leq 0 .
$$

Note that

$$
\dot{\theta}_{1}(t)=\Omega_{1}+\frac{K}{N}\left[\sum_{j \in \mathcal{C}_{1}} \sin \left(\theta_{j}-\theta_{1}\right)+\sum_{k=2}^{\ell-1} \sum_{j \in \mathcal{C}_{k}} \sin \left(\theta_{j}-\theta_{1}\right)+\sum_{j \in \mathcal{C}_{\ell}} \sin \left(\theta_{j}-\theta_{1}\right)\right] .
$$

Thus, we use

$$
\lim _{t \rightarrow \infty}\left(\theta_{j}-\theta_{1}\right)(t)=0, \quad j \in \mathcal{C}_{1}, \quad \text { and } \quad \lim _{t \rightarrow \infty}\left(\theta_{j}-\theta_{1}\right)(t)=-\frac{\pi}{2}, \quad j \in \mathcal{C}_{\ell}
$$

to derive the following relation:

$$
0 \leq \lim _{k \rightarrow \infty} \dot{\theta}_{1}\left(t_{n_{k}}\right)=\Omega_{1}-\frac{K}{N} \sum_{k=2}^{\ell-1}\left|\mathcal{C}_{k}\right| \cos \tilde{\theta}_{k}^{\infty}-\frac{K}{N}\left|\mathcal{C}_{\ell}\right|,
$$

where $\left|\mathcal{C}_{k}\right|$ denotes the cardinality of the $\operatorname{set} \mathcal{C}_{k}$, i.e.,

$$
\sum_{k=1}^{\ell}\left|\mathcal{C}_{k}\right|=N
$$

Similarly, we also obtain

$$
0 \geq \lim _{k \rightarrow \infty} \dot{\theta}_{N}\left(t_{n_{k}}\right)=\Omega_{N}+\frac{K}{N} \sum_{k=2}^{\ell-1}\left|\mathcal{C}_{k}\right| \sin \tilde{\theta}_{k}^{\infty}+\frac{K}{N}\left|\mathcal{C}_{1}\right| .
$$

We now combine (3.6), (3.7), and $\Omega_{1}-\Omega_{N}=D(\Omega)=K$ to derive

$$
\begin{aligned}
0 & \leq \lim _{k \rightarrow \infty} \dot{\theta}_{1}\left(t_{n_{k}}\right)-\lim _{k \rightarrow \infty} \dot{\theta}_{N}\left(t_{n_{k}}\right) \\
& =D(\Omega)-\frac{K}{N} \sum_{k=2}^{\ell-1}\left|\mathcal{C}_{k}\right|\left(\sin \tilde{\theta}_{k}^{\infty}+\cos \tilde{\theta}_{k}^{\infty}\right)-\frac{K}{N}\left(\left|\mathcal{C}_{1}\right|+\left|\mathcal{C}_{\ell}\right|\right) .
\end{aligned}
$$

Again, we use $K=D(\Omega)$ to yield

$$
\begin{aligned}
\frac{K}{N} \sum_{k=2}^{\ell-1}\left|\mathcal{C}_{k}\right|\left(\sin \tilde{\theta}_{k}^{\infty}+\cos \tilde{\theta}_{k}^{\infty}\right) & \leq D(\Omega)-\frac{K}{N}\left(\left|\mathcal{C}_{1}\right|+\left|\mathcal{C}_{\ell}\right|\right)=\frac{K}{N} \sum_{k=2}^{\ell-1}\left|\mathcal{C}_{k}\right| \\
& \leq \frac{K}{N} \sum_{k=2}^{\ell-1}\left|\mathcal{C}_{k}\right| .
\end{aligned}
$$


Since $\ell(\Omega) \geq 3$ and by Lemma A.2 (ii), we have

$$
\sum_{k=2}^{\ell-1}\left|\mathcal{C}_{k}\right|<\sum_{k=2}^{\ell-1}\left|\mathcal{C}_{k}\right|\left(\sin \tilde{\theta}_{k}^{\infty}+\cos \tilde{\theta}_{k}^{\infty}\right) \leq \sum_{k=2}^{\ell-1}\left|\mathcal{C}_{k}\right| .
$$

This provides the contradiction and yields the desired result.

Remark 3.2. Note that the contradiction in Part B is due to the fact that

$$
\sum_{k=2}^{\ell-1}\left|\mathcal{C}_{k}\right|>0
$$

which only makes sense for $\ell(\Omega) \geq 3$. Indeed, when $\ell(\Omega)=2$, it follows from [12] that

$$
\sup _{t>0} D(\theta(t))=\frac{\pi}{2}
$$

\section{Exponential complete synchronization}

In this section, we provide the proof of Theorem 2.2.

Since

$$
\left|\dot{\theta}_{i}(t)-\dot{\theta}_{j}(t)\right| \leq D(\omega(t)),
$$

it suffices to show that $D(\omega(t))$ converges to zero exponentially fast to obtain the desired result. To show this, we will derive a Gronwall type inequality for $D(\omega)$ :

$$
\frac{d}{d t} D(\omega(t)) \leq-K\left(\cos D_{\theta}^{\infty}\right) D(\omega(t)), \quad \text { a.e. } t .
$$

Derivation of (4.1): Basically, we follow the same arguments stated in [11] to derive the Gronwall inequality (4.1) for $D(\omega)$. To do this, we set

$$
\omega_{M}:=\max _{1 \leq i \leq N} \omega_{i}, \quad \omega_{m}:=\min _{1 \leq i \leq N} \omega_{i}
$$

Case A (Maximal frequency fluctuation): Since $\omega_{M}$ is Lipschitz continuous, it is differentiable in time $t$ almost everywhere. Next, using the same arguments in the case of $\theta_{M}$ in the proof of Proposition 3.3, it follows that there exist at most a countable number of times $0:=t_{0}<t_{1}<t_{2}<\cdots$ such that

$\omega_{M}$ is differentiable in the time interval $\left(t_{k-1}, t_{k}\right), \quad k=1,2, \cdots$.

For a given time zone $\left(t_{k-1}, t_{k}\right), \quad k=1, \cdots$, we choose an index $i$ such that

$$
\omega_{i}(t)=\omega_{M}(t), \quad t \in\left(t_{k-1}, t_{k}\right) .
$$

We use the result of Proposition 3.2,

$$
\left|\theta_{j}(t)-\theta_{i}(t)\right| \leq D(\theta(t)) \leq D_{\theta}^{\infty}<\frac{\pi}{2}
$$

to get

$$
\cos \left(\theta_{j}(t)-\theta_{i}(t)\right) \geq \cos D_{\theta}^{\infty}, \quad t \in\left(t_{k-1}, t_{k}\right) .
$$


We differentiate the system (1.1) with respect to $t$ and use (4.2) to find

$$
\begin{aligned}
\frac{d \omega_{i}}{d t} & =\frac{K}{N} \sum_{j=1}^{N} \cos \left(\theta_{j}-\theta_{i}\right)\left(\omega_{j}-\omega_{i}\right) \\
& \leq \frac{K \cos D_{\theta}^{\infty}}{N} \sum_{j=1}^{N}\left(\omega_{j}-\omega_{i}\right) \\
& =-K\left(\cos D_{\theta}^{\infty}\right) \omega_{i}, \quad t \in\left(t_{k-1}, t_{k}\right),
\end{aligned}
$$

where we used the following relations:

$$
\left(\omega_{j}-\omega_{i}\right) \leq 0, \quad \sum_{j=1}^{N} \omega_{j}=\sum_{i=1}^{N} \dot{\theta}_{i}=\sum_{i=1}^{N} \Omega_{i}+\frac{K}{N} \sum_{i, j=1}^{N} \sin \left(\theta_{j}-\theta_{i}\right)=\sum_{j=1}^{N} \Omega_{j}=0 .
$$

Thus, we have

$$
\frac{d \omega_{M}}{d t} \leq-K\left(\cos D_{\theta}^{\infty}\right) \omega_{M}, \quad \text { a.e.t. }
$$

Case B (Minimal frequency fluctuation): In this case, we apply the same argument as that used in Case A to find

$$
\frac{d \omega_{m}}{d t} \geq-K\left(\cos D_{\theta}^{\infty}\right) \omega_{m}, \quad \text { a.e. } t .
$$

Finally, we combine Case A and Case B to obtain the desired Gronwall's inequality. The standard Gronwall's inequality yields

$$
D(\omega(t)) \leq D(\omega(0)) \exp \left(-K\left(\cos D_{\theta}^{\infty}\right) t\right), \quad t \geq 0 .
$$

which denotes that complete frequency synchronization occurs asymptotically. This completes the proof of Theorem 2.2 (ii).

Next, we discuss the optimal speed of the relaxation of $D(\theta)$. To achieve this, we set

$$
E_{l}:=\sin \left(\theta_{M}-\theta_{m}\right)+\sin \left(\theta_{m}-\theta_{l}\right)+\sin \left(\theta_{l}-\theta_{M}\right) .
$$

If the asymptotic CFS of the Kuramoto model occurs, then for any $\varepsilon>0$ we have

$$
\left|E_{l}(t)-E_{l}^{\infty}\right| \leq \varepsilon, \quad t \geq T(\varepsilon) \quad \text { and } \quad E_{l}^{\infty}<0,
$$

where $E_{l}^{\infty}$ is given by the formula

$$
E_{l}^{\infty}:=\sin \left(\theta_{M}^{\infty}-\theta_{m}^{\infty}\right)+\sin \left(\theta_{m}^{\infty}-\theta_{l}^{\infty}\right)+\sin \left(\theta_{l}^{\infty}-\theta_{M}^{\infty}\right),
$$

and $E_{l}^{\infty}<0$ follows from Lemma A.2 (i). Therefore, asymptotically we find the following from (3.1):

$$
\dot{D}(\theta) \approx \underbrace{D(\Omega)\left(1-\frac{1}{N} \sum_{l \neq M, m}\left(\left|E_{l}^{\infty}\right|+\varepsilon\right)\right)}_{:=\Lambda\left(D(\Omega), E_{l}^{\infty}, \varepsilon\right)}-D(\Omega) \sin (D(\theta)), \quad t \gg 1 .
$$


Based on this observation, we have estimates for the optimal decay rate of $D(\theta)$.

Corollary 4.1. (Optimal speed of the relaxation of $D(\theta)$ ) Suppose that the framework $(\mathcal{F})$ holds, and let $\theta=\theta(t)$ be the solution to the system (1.1) - (1.3) with the initial data $\theta^{0} \in \mathcal{R}$. Thus, CFS occurs asymptotically and, more precisely, we have the following optimal convergence rates of $D(\theta(t))$ :

- Case A $\left(2 \tan ^{-1}\left[\frac{D(\Omega)-\sqrt{D(\Omega)^{2}-\Lambda^{2}}}{\Lambda}\right]-D(\theta(t))<0\right)$ :

$$
\begin{aligned}
& D(\theta(t)) \\
& \quad=2 \tan ^{-1}\left[\frac{1}{\Lambda}\left\{D(\Omega)-\sqrt{D(\Omega)^{2}-\Lambda^{2}} \tanh \left(\frac{1}{2}\left(t \sqrt{D(\Omega)^{2}-\Lambda^{2}}+C(T(\varepsilon))\right)\right)\right\}\right] .
\end{aligned}
$$

- Case B $\left(2 \tan ^{-1}\left[\frac{D(\Omega)-\sqrt{D(\Omega)^{2}-\Lambda^{2}}}{\Lambda}\right]-D(\theta(t))>0\right)$ :

$$
\begin{aligned}
& D(\theta(t)) \\
& \quad=2 \tan ^{-1}\left[\frac{1}{\Lambda}\left\{D(\Omega)-\sqrt{D(\Omega)^{2}-\Lambda^{2}} \operatorname{coth}\left(\frac{1}{2}\left(t \sqrt{D(\Omega)^{2}-\Lambda^{2}}+C(T(\varepsilon))\right)\right)\right\}\right]
\end{aligned}
$$

where

$$
C(T(\varepsilon))=\log \left|\frac{\Lambda \tan \frac{D(\theta(T(\varepsilon)))}{2}-D(\Omega)-\sqrt{D(\Omega)^{2}-\Lambda^{2}}}{\Lambda \tan \frac{D(\theta(T(\varepsilon)))}{2}-D(\Omega)+\sqrt{D(\Omega)^{2}-\Lambda^{2}}}\right|-T(\varepsilon) \sqrt{D(\Omega)^{2}-\Lambda^{2}} .
$$

Proof. We first obtain the asymptotic CFS of the Kuramoto oscillators from Theorem 2.2. Then, it follows from (4.3) and Case 1 in Lemma A.1 that

$$
\begin{aligned}
& t \sqrt{D(\Omega)^{2}-\Lambda^{2}}-T(\varepsilon) \sqrt{D(\Omega)^{2}-\Lambda^{2}} \\
& \quad \approx \log \left|\frac{\Lambda \tan \frac{D(\theta(t))}{2}-D(\Omega)-\sqrt{D(\Omega)^{2}-\Lambda^{2}}}{\Lambda \tan \frac{D(\theta(t))}{2}-D(\Omega)+\sqrt{D(\Omega)^{2}-\Lambda^{2}}}\right| \\
& \quad-\log \left|\frac{\Lambda \tan \frac{D(\theta(T(\varepsilon)))}{2}-D(\Omega)-\sqrt{D(\Omega)^{2}-\Lambda^{2}}}{\Lambda \tan \frac{D(\theta(T(\varepsilon)))}{2}-D(\Omega)+\sqrt{D(\Omega)^{2}-\Lambda^{2}}}\right| .
\end{aligned}
$$

Next, we divide the equation (4.6) into two cases A and B. By simple calculations, we obtain the desired results.

\section{Numerical simulations}

In this section, we provide several numerical results for the relaxation behavior of the phase and frequency diameters. In all of the numerical simulations, we used the fourth order Runge-Kutta method with $N=100$. It follows from [6] that the coupling strength $K$ should be larger than

$$
K_{l}:=\frac{N D(\Omega)}{2(N-1)}
$$

for the CFS, i.e., CFS does not occur for $K \leq K_{l}$. Of course, this does not mean that the CFS occurs for $K>K_{l}$. Therefore, the analytical results for CFS included in this paper are available for the regime $K \geq D(\Omega)$. Thus, it is not clear what will happen with $K \in\left(K_{l}, D(\Omega)\right)$ from the CFS perspective. Below, we address this issue using several numerical simulations. 


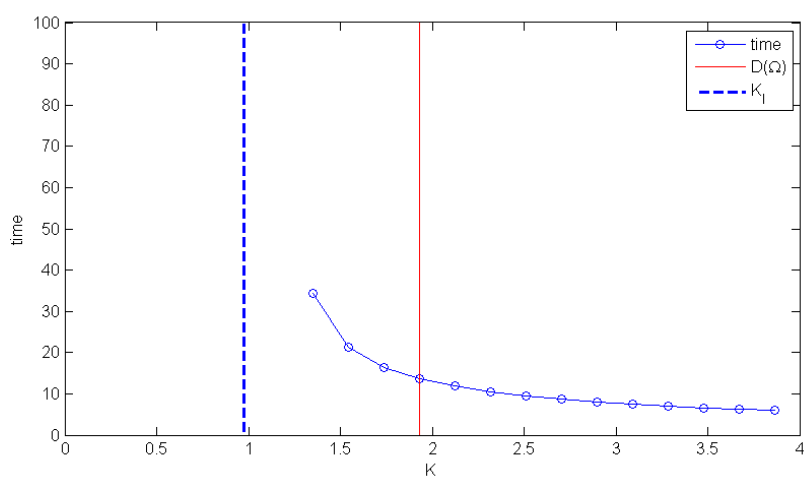

(a)
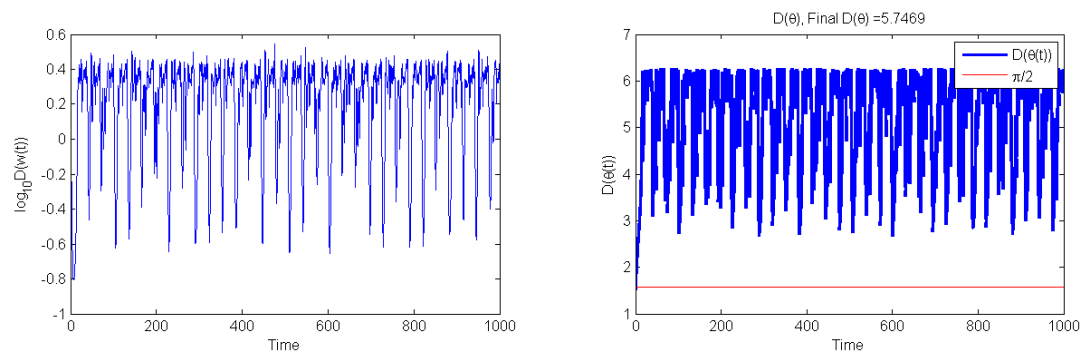

(b) $K_{l}<K<D(\Omega)$
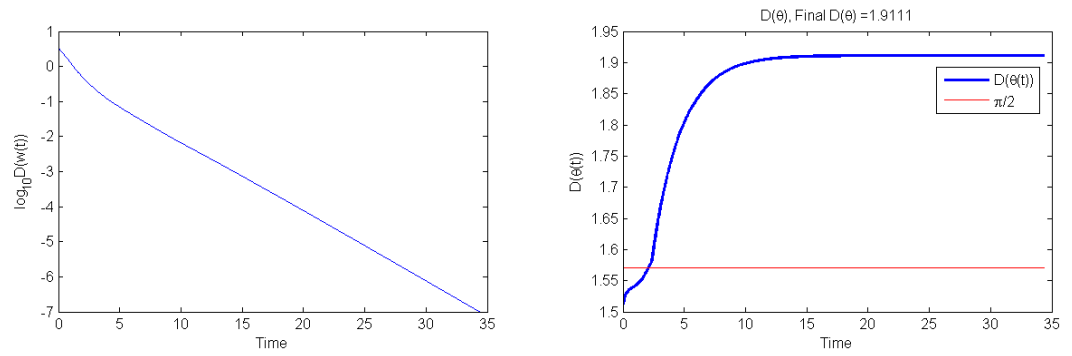

(c) $K_{l}<K<D(\Omega)$
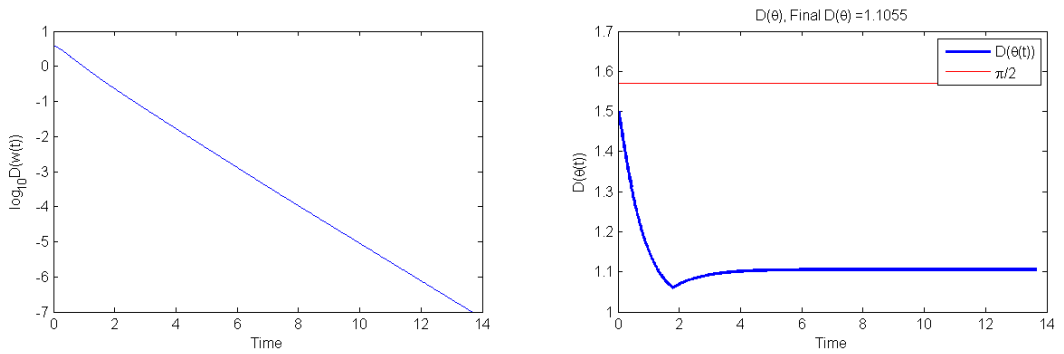

(d) $K=D(\Omega)$

Fig. 5.1: $\ell(\Omega)=100$ : (a) The time for the CFS versus the coupling strength $K$. (b) The behavior of $D(\omega(t))$ on a log-scale and $D(\theta(t))$ when $K_{l}<K=1.1590<D(\Omega)$. (c) The behavior of $D(\omega(t))$ on a log-scale and $D(\theta(t))$ when $K_{l}<K=1.3521<D(\Omega)$. (d) The behavior of $D(\omega(t))$ on a log-scale and $D(\theta(t))$ when $K=D(\Omega)$. 
In the simulations shown in figure 5.1, the natural frequencies were chosen randomly from a uniform distribution of $[-1,1]$ so that

$$
\ell(\Omega)=100 \quad \text { and } \quad D(\Omega)=1.9316,
$$

and the initial phase configuration was also selected uniformly at random from the interval $\left[0, \frac{\pi}{2}\right]$, so that

$$
D\left(\theta^{0}\right)=1.5336
$$

In figure 5.1, we varied $K$ from 0 to $2 D(\Omega)$ with the increment $\frac{D(\Omega)}{10}$.

In figure $5.1(\mathrm{a})$, we took the time where the frequency diameter was less than $10^{-7}$ by varying the coupling strength $K>K_{l}=0.9756$. Using these settings, we observed that the CFS did not occur when

$$
K \leq 1.1590 \text {. }
$$

In figure 5.1.(b), we can see that the frequency diameter $D(\omega(t))$ did not converge to zero when

$$
K_{l}<K=1.1590<D(\Omega) .
$$

In contrast, figure 5.1.(c) shows that $D(\omega(t))$ decays exponentially to zero, although the limit of $D(\theta(t))$ was higher than $\frac{\pi}{2}$ for $K=1.3521(<D(\Omega))$. Thus, if there is a coupling strength $K$ such that the frequency diameter converges algebraically, it should lie between 1.1590 and 1.3521 .

In figure $5.1(\mathrm{~d}), D(\omega)$ converges to zero exponentially for $K=D(\Omega)$ and

$$
D^{\infty}=1.1055<\frac{\pi}{2} .
$$

This is consistent with the analytic result in Theorem 2.2 (i).

\section{Conclusion}

In this paper, we provided an admissible class of initial configurations that led to the exponential synchronization of the Kuramoto model at the coupling strength $K=$ $D(\Omega)$. In the special case where Kuramoto's ensemble consists of two distinct natural frequencies, Ha and Kang [12] showed that asymptotic CFS occurs algebraically at the order of $(1+t)^{-1}$ given the initial configurations in $\mathcal{R}$. In contrast, when $\ell(\Omega) \geq 3$, we showed that the relaxation rate to the phase-locked states was at least exponentially fast. It would be very interesting to find the coupling strength $K$ that guarantees a slow relaxation (algebraic decay rate) for some classes of initial phase configurations. We will leave this interesting issue to future work.

Acknowledgment. The work of S.-Y. Ha was supported by the Basic Science Research Program through a National Research Foundation of Korea (NRF-201100015388), and the work of M. Kang was supported by the Basic Science Research Program (NRF 2012-001799).

Appendix A. Elementary estimates. In this section, we present several elementary estimates that are used in the proof of CFS.

Consider the Adler equation:

$$
\dot{y}=\Omega-K \sin y, \quad t>0, \quad y(0)=y_{0},
$$


where $\Omega$ and $K$ are positive constants. In the following lemma, we explicitly present the implicit form of the solution to equation (A.1).

Lemma A.1. [5] The Adler equation has a global solution that satisfies

(i) Case $1(0 \leq \Omega<K)$ :

$$
t \sqrt{K^{2}-\Omega^{2}}=\log \left|\frac{\Omega \tan \frac{y(t)}{2}-K-\sqrt{K^{2}-\Omega^{2}}}{\Omega \tan \frac{y(t)}{2}-K+\sqrt{K^{2}-\Omega^{2}}}\right|-\log \left|\frac{\Omega \tan \frac{y_{0}}{2}-K-\sqrt{K^{2}-\Omega^{2}}}{\Omega \tan \frac{y_{0}}{2}-K+\sqrt{K^{2}-\Omega^{2}}}\right| .
$$

(ii) Case $2(\Omega=K)$ :

$$
y(t)=2 \arctan \left[\frac{2 \tan \frac{y_{0}}{2}+\Omega\left(1-\tan \frac{y_{0}}{2}\right) t}{2+\Omega\left(1-\tan \frac{y_{0}}{2}\right) t}\right] .
$$

(iii) Case $3(K<\Omega)$ :

$$
\begin{aligned}
y(t) & =2 \arctan \left[\frac { 1 } { R ^ { \infty } } \left\{\sqrt{\left(R^{\infty}\right)^{2}-1}\right.\right. \\
& \left.\left.\times \tan \left\{\frac{K t}{2} \sqrt{\left(R^{\infty}\right)^{2}-1}+\tan ^{-1}\left(\frac{R^{\infty} \tan \frac{y_{0}}{2}-1}{\sqrt{\left(R^{\infty}\right)^{2}-1}}\right)\right\}+1\right\}\right], \quad R^{\infty}:=\frac{\Omega}{K} .
\end{aligned}
$$

Lemma A.2. The following elementary estimates hold.

(i) Let $\theta_{i}, i=1,2,3$ be the three values in $[-\pi, \pi)$ that satisfy

$$
\theta_{i} \leq 0, i=1,2 \quad \theta_{1}+\theta_{2}+\theta_{3}=0 .
$$

Thus, we have

$$
\sin \theta_{1}+\sin \theta_{2}+\sin \theta_{3} \leq 0
$$

where the equality holds if and only if $\theta_{1} \theta_{2}=0$.

(ii) For $\theta \in\left[0, \frac{\pi}{2}\right]$,

$$
\sin \theta+\cos \theta \geq 1
$$

and the equality holds if and only if $\theta=0$ or $\theta=\frac{\pi}{2}$.

Proof.

(i) We use the elementary properties of trigonometric functions to show that

$$
\begin{aligned}
\sin \theta_{1}+\sin \theta_{2}+\sin \theta_{3} & =\sin \theta_{1}+\sin \theta_{2}-\sin \left(\theta_{1}+\theta_{2}\right) \\
& =\sin \theta_{1}\left(1-\cos \theta_{2}\right)+\sin \theta_{2}\left(1-\cos \theta_{1}\right) \\
& \leq 0, \quad\left(\because \sin \theta_{1}, \sin \theta_{2} \leq 0\right) .
\end{aligned}
$$

Note that the equality holds if and only if

$$
\sin \theta_{1}\left(1-\cos \theta_{2}\right)=0 \quad \sin \theta_{2}\left(1-\cos \theta_{1}\right)=0 \quad \Longleftrightarrow \quad \theta_{1} \theta_{2}=0 .
$$

(ii) Since $\theta+\frac{\pi}{4} \in\left[\frac{\pi}{4}, \frac{3 \pi}{4}\right]$, it is easy to see that

$$
\sin \theta+\cos \theta=\sqrt{2} \sin \left(\theta+\frac{\pi}{4}\right) \geq \sqrt{2} \frac{1}{\sqrt{2}}=1, \quad \theta \in\left[0, \frac{\pi}{2}\right],
$$

and the equality holds if and only if $\theta=0, \frac{\pi}{2}$. 


\section{REFERENCES}

[1] J.A. Acebron, L.L. Bonilla, C.J.P. Pérez Vicente, F. Ritort, and R. Spigler, The Kuramoto model: A simple paradigm for synchronization phenomena, Rev. Mod. Phys., 77, 137-185, 2005.

[2] J. Buck and E. Buck, Biology of synchronous flashing of fireflies, Nature, 211, 562, 1966.

[3] N.J. Balmforth and R. Sassi, A shocking display of synchrony, Physica D, 143, 21-55, 2000.

[4] Y. Choi, S.Y. Ha, and S.B. Yun, Complete synchronization of Kuramoto oscillators with finite inertia, Physica D, 240, 32-44, 2010.

[5] Y. Choi, S.Y. Ha, S.E. Jung, and Y. Kim, Asymptotic formation and orbital stability of phaselocked states for the Kuramoto model, Physica D, 241, 735-754, 2012.

[6] N. Chopra and M.W. Spong, On exponential synchronization of Kuramoto oscillators, IEEE Trans. Automatic Control, 54, 353-357, 2009.

[7] F. de Smet and D. Aeyels, Partial frequency in finite Kuramoto-Sakaguchi model, Physica D, 234, 81-89, 2007.

[8] F. Dörfler and F. Bullo, On the critical coupling for Kuramoto oscillators, SIAM J. Appl. Dyn., 10, 1070-1099, 2011.

[9] F. Dörfler and F. Bullo, Synchronization and transient stability in power networks and nonuniform Kuramoto oscillator, Amer. Cont. Conf., Baltimore, 930-937, 2010.

[10] G.B. Ermentrout, Synchronization in a pool of mutually coupled oscillators with random frequencies, J. Math. Biol., 22, 1-9, 1985.

[11] S.Y. Ha, T. Ha, and J.H. Kim, On the complete synchronization for the globally coupled Kuramoto model, Physica D, 239, 1692-1700, 2010.

[12] S.Y. Ha and M. Kang, Fast and slow relaxations to bi-cluster configurations for the ensemble of Kuramoto oscillators, Quarterly of Appl. Math., to appear.

[13] S.Y. Ha, C. Lattanzio, B. Rubino, and M. Slemrod, Flocking and synchronization of particle models, Quarterly of Appl. Math., 69, 91-103, 2011.

[14] A. Jadbabaie, N. Motee, and M. Barahona, On the stability of the Kuramoto model of coupled nonlinear oscillators, in: Proceedings of the American Control Conference, Boston, Massachusetts, 2004.

[15] Y. Kuramoto, Chemical Oscillations, Waves and Turbulence, Springer-Verlag, Berlin, 1984.

[16] Y. Kuramoto, International symposium on mathematical problems in mathematical physics, Lecture Notes in Theoretical Physics, 30, 420, 1975.

[17] R.E. Mirollo and S.H. Strogatz, The spectrum of the locked state for the Kuramoto model of coupled oscillator, Physica D, 205, 249-266, 2005.

[18] R.E. Mirollo and S.H. Strogatz, The spectrum of the partially locked state for the Kuramoto model of coupled oscillator, J. Nonlin. Sci., 17, 309-347, 2007.

[19] C.S. Peskin, Mathematical Aspects of Heart Physiology, Courant Inst. Math. Sci., New York, 1975.

[20] A. Pikovsky, M. Rosenblum, and J. Kurths, Synchronization: A Universal Concept in Nonlinear Sciences, Cambridge University Press, Cambridge, 2001.

[21] J.H. Sheeba, V.K. Chandrasekar, A. Stefanovska, and P.V.E. McClintock, Routes to synchrony between asymmetrically interacting oscillator ensembles, Phys. Review E., 78, 025201(R), 2008.

[22] S.H. Strogatz, From Kuramoto to Crawford: Exploring the onset of synchronization in populations of coupled oscillators, Physica D, 143, 1, 2001.

[23] S.H. Strogatz and R.E. Mirollo, Stability of incoherence in a population of coupled oscillators, J. Stat. Phys., 63, 613-635, 1991.

[24] S.H. Strogatz, R.E. Mirollo, and P.C. Mattews, Coupled nonlinear oscillators below the synchronization threshold: Relaxation by generalized Landau damping, Phys. Rev. Lett., 68, 2730-2733, 1992.

[25] J.L. van Hemmen and W.F. Wreszinski, Lyapunov function for the Kuramoto model of nonlinearly coupled oscillators, J. Stat. Phys., 72, 145-166, 1993.

[26] M. Verwoerd and O. Mason, Global phase-locking in finite populations of phase-coupled oscillators, SIAM J. Appl. Dyn., 7, 134-160, 2008.

[27] M. Verwoerd and O. Mason, On computing the critical coupling coefficient for the Kuramoto model on a complete bipartite graph, SIAM J. Appl. Dyn., 7, 134-160, 2008.

[28] S. Watanabe and S.H. Strogatz, Constants of motion for superconducting Josephson arrays, Physica D, 74, 197-253, 1994.

[29] A.T. Winfree, The Geometry of Biological Time, Springer, New York, 1980.

[30] A.T. Winfree, Biological rhythms and the behavior of populations of coupled oscillators, J. Theor. Biol., 16, 15-42, 1967. 